\title{
Efficacy of Tripterygium Glycosides for Diabetic Nephropathy: A Meta-Analysis of Randomized Controlled Trials
}

Huabin Guo Huabin Guo

Yangtze University Medical School https://orcid.org/0000-0002-6799-3110

Hua-Bin Guo

Yangtze university

Jia-Qing Peng ( $\sim$ pjq5008@163.com)

Yangtze University Health Science Center

Ke-Kai Zhang

Yangtze University Medical School

Guang-Zhi Zhong

Yangtze University Medical School

Wei-Hong Chen

Hanyin

Gui-Xin Shi

Hanyin

\section{Research article}

Keywords: Tripterygium glycosides, Diabetic nephropathy, randomized controlled trials, Meta-analysis

Posted Date: February 9th, 2021

DOl: https://doi.org/10.21203/rs.3.rs-194899/v1

License: (9) (i) This work is licensed under a Creative Commons Attribution 4.0 International License.

Read Full License 


\section{Abstract}

Backgrounds: Diabetic nephropathy (DN) is one of the most important clinical complications of diabetes mellitus (DM) and is the most common cause of end-stage renal disease. Currently, there is no highly effective medicine that can prevent, halt, or reverse the progressive course of DN. Initial clinical data showed that Tripterygium glycosides (TGs), a traditional Chinese medicine, can decrease proteinuria in patients with DN.

Objectives: The objective of the present study is to investigate the efficacy and safety of TGs for the treatment of DN by meta-analysis of randomized controlled trials (RCTs).

Methods: All RCTs of TGs for DN were collected from The China National Knowledge Infrastructure (CNKI), Web of Science, Wanfang Data, Chinese Biomedical Literature Database (CBM), China Science and Technology Journal Database (VIP) by setting the study inclusion and elimination standards. Two reviewers evaluated the quality of the trials and extracted the data independently. RevMan 5.4 software was used for meta-analyses. The primary outcome was a change in 24-hours urinary total protein (24h TUP).

Results: 26 RCTs with 1824 participants were collected for detailed evaluation. The meta-analysis results indicated that TGs was compared with the controls, TGs showed significant effects in reducing 24h TUP $[\mathrm{WMD}=-0.84,95 \% \mathrm{Cl}(-1.09,-0.59)]$, elevating serum albumin [WMD $=2.88,95 \% \mathrm{Cl}(1.87,3.90)]$, and the total efficiency [OR $=4.08,95 \% \mathrm{Cl}(2.37,7.04)]$. This effect was consistent across the subgroups of period of intervention.

Conclusions: The present research showed that TGs was significantly associated with improvement of renal function in patients with DN. TGs offers a novel way in treating DN, more high quality RCTs are needed for better understanding of the role of TGs in DN therapy.

\section{Background}

Diabetic nephropathy(DN) is one of the most important clinical complications of diabetes mellitus (DM) and is the most common cause of end-stage renal disease ${ }^{[1]}$. pathological changes of clinical DN patients are characterized by glomerular mesangial matrix expansion, glomerular basement membrane (GBM) thickening, and formation of glomerular nodular sclerosis ${ }^{[2-3]}$. The clinical manifestations of DN include hypertension, massive proteinuria, edema, and progressive decrease in renal function ${ }^{[4]}$. The precise mechanisms of DN pathogenesis are yet to be fully elucidated. The studies were from more recent years show that inflammation played a vital role in the pathogenesis of $\mathrm{DN}^{[5]}$, It is believed that persistent hyperglycemia plays a positive role in the pathogenesis of $\mathrm{DN}$, but simply controlling blood glucose doesn't stop the progression of DN symptoms ${ }^{[6]}$. How to select new drugs for diabetic nephropathy from the perspective of anti-inflammatory and immunosuppressive is a subject for kidney disease workers. 
Tripterygium wilfordii is a traditional Chinese medicine, its extract Tripterygium glycosides (TGs) belong

to non-steroidal immuno suppressants, can effectively inhibit cellular and humoral immunity ${ }^{[7,8]}$. In 1977 , Chinese scholars first reported its role in reducing proteinuria in glomerulonephritis, and TGs began to be used in clinical treatment of renal diseases. TGs has been widely used in the treatment of primary glomerulonephritis and immune-related nephritis with accurate curative effect ${ }^{[9]}$. At present, some clinical studies apply TGs in the treatment of DN, It is suggested that TGs can antagonize the anti-inflammatory effect in the pathogenesis of DN and thus protect renal function ${ }^{[10]}$. Therefore, this study systematically evaluated the efficacy and safety of TGs in the treatment of DN on the basis of existing RCTs, Aimed at providing evidence-based medical evidence for the clinical application of TGs in the treatment of DN.

\section{Methods}

\subsection{Search strategy}

We searched RCTs via PubMed, Medline, Embase,China National Knowledge Infrastructure (CNKI), Web of Science, Wanfang Data, Chinese Biomedical Literature Database (CBM), China Science and Technology Journal Database (VIP) up to June 2020. The following keywords and Medical Subject Heading (MeSH) were searched: "diabetic kidney disease" or "diabetic nephropathies" or "diabetic glomerulosclerosis", and "Tripterygium wilfordii Hook" or "tripterygium glycosides". At the same time, the reference lists of included studies, review articles, and reports of academic congresses were checked manually, in order to prevent from neglecting any relevant studies.

\subsection{Inclusion and exclusion criteria}

Included criteria: Trials were considered to be eligible for inclusion if they met all of the following criteria: (1) Research type: studies based on RCTs. (2) Research subjects: the patients of the original studies have been clinically diagnosed with DN. (3) Interventions: The control group was treated with conventional treatment and the experimental group was added with TGs on the basis of the control group. Other measures were consistent in the two groups. (4) Outcome measures: 24 hours urinary total protein (24 h UTP), serum creatinine (SCr), blood urea nitrogen (BUN), creatinine clearance (Ccr), albumin (Alb), alanine aminotransferase (ALT), white blood cells (WBC). Elevated ALT is a serological manifestation of liver damage, and reduced WBC is a serological manifestation of myelosuppression. liver function damage and myelosuppression are considered to be major adverse reactions. total efficiency was defined as obvious effect plus effective according to guidelines for clinical research of new chinese medicine drugs $^{[11-12]}$.

Exclusion criteria: (1) Non-randomized controlled controlled trials. (2) studies of patients were not clearly diagnosed or patients with DN, acute and chronic nephritis, and other causes of hypoproteinemia. (3) Other Chinese medicines being used in the control group or the experimental group. (4) studies such as systemic reviews, meta-analysis, casereports, animal experimental studies, etc. Lack of rigorous experimental design, inappropriate statistical method or lack of related outcome measures. 


\subsection{Data extraction}

Data of each included study was extracted independently and cross checked by two investigators (GuiXin Shi, Xuan Wang). including study design, randomization, diagnostic criteria, the first author's name, publication year, sample size, treatment duration, outcomes. Disagreements and differences in inclusion of studies were dealt with by consensus or discussion with a third researchers (Ke-Kai Zhang). All of reference lists of researches that had been identified were checked out carefully again to prevent the omissions and the details of the selection process are shown in Fig. 1.

\subsection{Quality assessment}

We evaluated the quality of studies using the Review Manager (vision 5.4.1) risk-of-bias tool. The tool includes the following 7 sections: random sequence generation (selection bias), allocation concealment (selection bias), blinding of participants and personnel (performance bias), blinding of outcome assessment (detection bias), incomplete outcome data (attrition bias), selective reporting (reporting bias) and other biases. (Fig. 2 and Supplemental Fig. 1 )

\subsection{Statistical analysis}

We used the Review Manager(version 5.4.1) and STATA (version 12.0, Stata SE) software for statistical analysis. Count data was summarized by forest plot, with expressed as odds ratio (OR) with $95 \%$ confidence interval $(\mathrm{Cl})$. Measurement data was summarized by forest plot, with expressed as standard mean difference (SMD) and mean difference (MD) with 95\% confidence interval $(\mathrm{Cl})$. We used $\mathrm{I}^{2}$ statistic to assess heterogeneity of included RCTs. The heterogeneity is graded according to $\mathrm{I}^{2}$, there are slow, moderate, and high heterogeneity. If $\mathrm{I}^{2}$ was lower than $50 \%$, the heterogeneity was considered acceptable and we used a fixed-effect model to analyze data. If $\mathrm{I}^{2}$ was higher than $50 \%$, sensitivity analysis and subgroup analysis to explore possible sources of heterogeneity. If heterogeneity remains higher than $50 \%$, we used a random-effect model to analyze data. Quantitative methods such as Begg's and Egger's test will be used to assess publication bias

\section{Results}

\section{1. study characteristics}

we selected and read 26 RCTs $^{[13-38]}$ in full to use for the meta-analysis. A total of 26 trials including 939 DN Patients receiving TGs and 885 controls that met our inclusion criteria were included in the present study and all cases were treated with routine treatment such as reduce blood glucose and bloodpress. All the studies were conducted in China. The characteristics of included studies are summarized in Supplemental Table 1.

\subsection{4-hours urinary total protein (24 h UTP)}


Twenty four trials ${ }^{[13-17,19-23,25-38]}$ demonstrated a difference in 24-hours urinary total protein (24 $\mathrm{h}$ UTP) between the experimental group and control group. The results were included in RevMan 5.4.1 software. Considering the significant heterogeneity $\left(P<0.05, I^{2}=85 \%\right)$, Therefore, the random effect model was adopted. The result of meta-analysis showed that TGs group is more effective at reducing $24 \mathrm{~h}$ UTP [WMD $=-0.84,95 \% \mathrm{Cl}(-1.09,-0.59)]$.

The subgroups were divided into $\mathrm{t}<3$ months, $\mathrm{t}<6$ months and $\mathrm{t}>6$ months of TGs compared to control treatment (Fig. 3). There was still obvious heterogeneity within each subgroup [t< 3 months: $\left(I^{2}=98 \%, P<\right.$ $0.05) ; 3<\mathrm{t}<6$ months: $\left(I^{2}=97 \%, P<0.05\right) ; \mathrm{t}>6$ months: $\left.\left(I^{2}=85 \%, P<0.05\right)\right]$. Subgroup analysis also showed that there was a statistically significant difference in $24 \mathrm{~h}$ UTP between the experimental group and control group, experiment group was superior to the control group in reducing the $24 \mathrm{~h} \mathrm{UTP}$ [ $\mathrm{t}<3$ months: $\mathrm{WMD}=-0.61,95 \% \mathrm{Cl}(-1.18,-0.03) ; 3<\mathrm{t}<6$ months: $\mathrm{WMD}=-0.61,95 \% \mathrm{Cl}(-1.12,-0.09) ; \mathrm{t}>6$ months: $\mathrm{WMD}=-1.06,95 \% \mathrm{Cl}(-1.35,-0.77)]$. The results revealed that it was more significantin reducing $24 \mathrm{~h}$ UTP for the experimental group compared to the control group when the course of treatment was $\mathrm{t} \geq$ 3 months.

The subgroup based on $24 \mathrm{~h}$ UTP baseline of inclusion criteria analysis showed there was still obvious heterogeneity within each subgroup $\left[>1.0 \mathrm{~g} / 24 \mathrm{~h}:\left(\mathrm{P}<0.05, \mathrm{I}^{2}=84 \%\right) ;>1.5 \mathrm{~g} / 24 \mathrm{~h}:\left(\mathrm{P}<0.05, \mathrm{l}^{2}=96 \%\right)\right.$; $\left.>2.5 \mathrm{~g} / 24 \mathrm{~h}:\left(\mathrm{P}<0.05, \mathrm{I}^{2}=59 \%\right) ;>3.5 \mathrm{~g} / 24 \mathrm{~h}:\left(\mathrm{P}<0.05, \mathrm{I}^{2}=85 \%\right)\right]$. Other subgroups indicated the combined group was superior to the control group in reducing the $24 \mathrm{~h}$ UTP $[>1.0 \mathrm{~g} / 24 \mathrm{~h}: \mathrm{WMD}=-0.51,95 \% \mathrm{Cl}(-0.72$, $-0.31) ;>1.5 \mathrm{~g} / 24 \mathrm{~h}: \mathrm{MD}=-1.22,95 \% \mathrm{Cl}(-1.76,-0.69) ;>3.5 \mathrm{~g} / 24 \mathrm{~h}: \mathrm{WMD}=-0.95,95 \% \mathrm{Cl}(-1.34,-0.56)]$. (Figure. 4).

\subsection{Serum albumin (Alb)}

The serum albumin (Alb) concentration is related to glomerular filtration rate, and its levels decline in DN patients. Seventeen trials ${ }^{[13-18,20,23,26-33]}$ reported a difference in Alb between the experimental and control group. Two trials with Alb over $40 \mathrm{~g} / \mathrm{L}$ after treatment was excluded ${ }^{[29,32]}$. Statistical heterogeneity analysis indicated significant heterogeneity across the studies $\left(I^{2}=67.0 \%, P<0.05\right)$.

Therefore, Result conducted by random-effect model showed the experimental group was superior to the control group in elevating Alb [WMD $=2.88,95 \% \mathrm{Cl}(1.87,3.90)]$. Subgroup analysis based on the course of treatment. All subgroups indicated the experimental group was superior to the control group in elevating Alb $[3 \leq \mathrm{t}<6$ months: $\mathrm{WMD}=3.02,95 \% \mathrm{Cl}(2.22,3.83) ; \mathrm{t}>6$ months: $\mathrm{WMD}=2.57,95 \% \mathrm{Cl}(0.59$, 4.54)]. (Figure. 5).

\subsection{Serum creatinine (SCr)}

twenty three trials ${ }^{[13-33,37-38]}$ demonstrated a difference in Serum creatinine (SCr) between the experiment and control group. Significant heterogeneity $\left(P<0.05, \mathrm{I}^{2}=78 \%\right)$ was identified and analyzed data by a random-effect model. Meta-analysis showed that TGs could improve SCr better than control 
group [WMD $=-4.77 ; 95 \% \mathrm{Cl}(-4.78,-1.75)]$ in DN patients. Subgroup analysis based on the course of treatment showed There was still significant heterogeneity within each subgroup [ $t<3$ months: $(P=$ $\left.0.06, I^{2}=65.0 \%\right) ; 3<t<6$ months: $\left(P<0.05, I^{2}=76.0 \%\right) ; t>6$ months: $\left.\left(P<0.05, I^{2}=83.0 \%\right)\right]$. Subgroup analysis showed there was no difference in $\mathrm{SCr}$ between the experimental group and control group if treatment lasted less than 3 months and 6 months [ $\mathrm{t}<3$ months: WMD $=-4.37,95 \% \mathrm{Cl}(-10.04,1.30) ; 3<\mathrm{t}<$ 6 months: $\mathrm{WMD}=-5.14,95 \% \mathrm{Cl}(-11.29,1.00)]$. but there was a significant difference in SCr between the experimental group and control group if the course of treatment more than 6 months (WMD $=-4.96,95 \%$ Cl (-9.80, -0.11). (Fig. 6).

\subsection{Blood urea nitrogen (BUN)}

Blood urea nitrogen (BUN) also is an indicator of kidney function, often used to reflect the change of kidney function in DN patients. 9 studies ${ }^{[15-16,21-23,26,29,33,37]}$ reported a difference in BUN between the experimental and the control group. The significant heterogeneity was found across these studies $(\mathrm{P}<$ $\left.0.05, I^{2}=79 \%\right)$. Therefore, the data was pooled by a random effect model. The result indicated that there was no significant difference in reducing BUN betwee TGs and routine treatment in DN patients [WMD = $0.37 ; 95 \% \mathrm{Cl}(-0.79,0.04) ; \mathrm{P}=0.08)]$. ( Fig. 7).

\subsection{Creatinine clearance (CCr)}

Creatinine clearance ( $\mathrm{CCr}$ ) as the main indicator of kidney function was reduced in DN Patients. Seven trials ${ }^{[17,24-25,28-29,31,38]}$ reported a difference in CCr between the experiment and control group. Statistical heterogeneity analysis indicated slow heterogeneity across the studies $\left(I^{2}=13.0 \%, P=0.33\right)$. Therefore, a fixed-effect model was used. The result showed TGs could improve CCr better than routine treatment $[(\mathrm{SMD}=0.49 ; 95 \% \mathrm{Cl}(0.27,0.70)]$. (Fig. 7).

\subsection{Adverse reactions}

Alanine aminotransferase (ALT) is Measurement of liver damage in TGs. The effect on ALT was mentioned in 10 studies $^{[15-16,18-19,28-29,31-32]}$ and significant heterogeneity was found across these studies $\left(P<0.05, I^{2}=66 \%\right)$. Therefore, the data was pooled by a random-effect model. The result indicated that the TGs did not cause ALT elevation compared to the control because there was no significant difference between the experimental and the control group $[\mathrm{WMD}=1.18,95 \% \mathrm{Cl}(-0.68,3.04)$, $P=0.21$. (Fig. 8).

The white blood cells (WBC) was mentioned in eight studies $[15,18,21,28-29,31-33]$ and significant heterogeneity was found across these studies $\left(P=0.01, I^{2}=62 \%\right)$. We conducted a sensitivity analysis by deleting a study ${ }^{[29]}$ eliminate heterogeneity $\left(P=0.29, I^{2}=18 \%\right)$. Therefore, a fixed-effect model was used. The result showed TGs could make reduction in WBC than routine treatment [(WMD $=-0.26 ; 95 \% \mathrm{Cl}(-0.38$, $-0.14)]$. (Fig. 9). 


\subsection{Total efficacy}

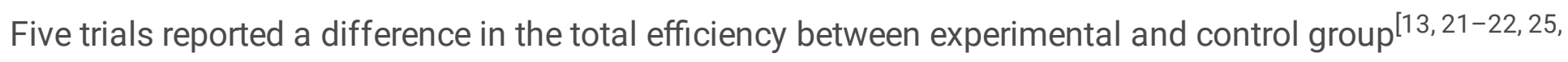
38]. There was a slow heterogeneity in the total efficiency $\left(I^{2}=7.0 \%, P=0.37\right)$. Thus, experimental odds ratio (OR) was pooled by a fixed-effect model. The total efficiency of the experimental group was obviously higher than the control $[\mathrm{OR}=4.08,95 \% \mathrm{Cl}(2.37,7.04)]$.

\subsection{Sensitivity analysis and publication bias}

Sensitivity analysis can assess the stability and reliability of the conclusions of the meta analysis. sensitivity analysis with $24 \mathrm{~h}$ UTP and SCr in Stata 12.0 software. The results illustrated that the metaanalysis about $24 \mathrm{~h}$ UTP and SCr had low sensitivity and high stability in analysis of patients with DN. (Supplemental Fig. 2 and Fig. 3). Qualitative analysis of the funnel plot and graph symmetry was used to assess publication bias. The publication bias of $24 \mathrm{~h}$ UTP and SCr for included RCTs was evaluated by comparing the symmetry of the funnel plot. (Supplemental Fig. 4 and Fig. 5). Begg's and Egger's test with $24 \mathrm{~h}$ UTP and SCr in Stata 12.0 to further confirm whether the publication bias for $24 \mathrm{~h}$ UTP and SCr. The result to $24 \mathrm{~h}$ UTP, Egger $(\mathrm{t}=-1.95, \mathrm{P}>0.05)$ and Begg test $(\mathrm{Pr}>|\mathrm{Z}|=0.47)$. The result to SCr, Egger $(\mathrm{t}=-$ $0.92, P>0.05)$ and Begg test $(P r>|Z|=1.00)$. which indicated that there was no evidence of substantial publication bias. However, it is needed to highlight that the Quality of of RCTs included restricted this application.

\section{Discussion}

Diabetic nephropathy is a progressive kidney disease caused by damage to the capillaries in the kidneys' glomeruli ${ }^{[39]}$. DN is a leading cause of end-stage renal disease worldwide ${ }^{[40]}$. The pathogenesis of diabetic nephropathy includes many factors such as hemodynamics, Inflammation, immune reactions, oxidative stress, podocytes injury ${ }^{[40-41]}$. There are still no effective treatments for $\mathrm{DN}$, the purposes for treatment for DN were to decrease the progression of kidney damage and control associated complications such as proteinuria, hypoproteinemia, and others. The major treatment modality of diabetic nephropathy was angiotensin converting enzyme inhibitor/angiotensin receptor blockers $(\mathrm{ARB} / \mathrm{ACEI})^{[42]}$. Treatment with ARB/ACEI can generally reduce proteinuria and alleviate the progression of kidney injury. Other methods include controlling blood pressure, managing blood sugar, exercise, and a low-protein diet are used to treat DN, partly retarding the progression of DN. TGs is widely used to treat primary glomerulonephritis and immune-related nephritis. Recently years, several prospective clinical studies have discussed the clinical efficacy of TGs in treatment of DN. Most of the studies showed that TGs can improve clinical efficacy, decrease the $24 \mathrm{~h}$ urinary protein, and serum creatinine. In DN, TGs not only improved proteinuria, but also alleviated kidney pathological changes and reduced the inflammation levels of kidney via p38 mapk pathway ${ }^{[43-44]}$. 
In our present meta-analysis, 26 clinical studies including 1824 subjects (939 in experiment group and 885 in control group) were included. we found the TGs groups howed significant effects in reducing 24UTP $(P<0.05)$, elevating Alb $(P<0.05)$, reducing Scr $(P<0.05)$, elevating $\operatorname{Ccr}(P<0.05)$, and increasing the total efficiency, when compared to the control group, which is the same as others meta-analysis ${ }^{[11,45,46]}$. However, TGs treatment had no obvious advantage in improving BUN $(P>0.05)$ and increasing ALT $(P>$ 0.05), The adverse effects of bone marrow suppression is obvious in TGs group, there was significant effect in reducing WBC $(P<0.05)$, when compared to the control group. The present evidence indicates that TGs can improve clinical efficacy, reduce the $24 \mathrm{~h}$ urinary protein, and serum creatinine as compared with routine treatment. Thus, We can speculate that compared with control group, TGs can repair the renal function of DN patients to a certain extent, but the pooled results showed the TGs associated toxicity of bone marrow suppression was significant higher than that of the control group.

There were Several limitations in this meta-analysis. (1) The general quality of the included studies was poor, most studies did not describe allocation concealment and binding, These are most likely to cause selection bias, attrition bias, and reporting bias; (2) The diagnostic criteria for DN patients are not uniform, which may lead to different stages of DN for patients included; (4) Only Chinese and English studies were included in this meta-analysis; (5) significant statistical heterogeneity still existed across the included studies and should be further explored.

\section{Abbreviations}

DN, Diabetic nephropathy; TGs, Tripterygium glycosides; RCTs, Randomized controlled trials; CNKI, China National Knowledge Infrastructure; CBM, Chinese Biomedical Literature Database; 24 h TUP, 24-hours urinary total protein; DM, Diabetes mellitus; GBM, Glomerular basement membrane; MeSH, Medical Subject Heading; SCr, Serum creatinine; BUN, Blood urea nitrogen; Ccr, Creatinine clearance; Alb, Albumin; ALT, Alanine aminotransferase; WBC, White blood cells.

\section{Declarations}

\section{Acknowledgements}

We would like to acknowledge Dr. Jia-Qing Peng for her suggestions in final editing of this manuscript.

\section{Funding}

No funding exists regarding this manuscript.

\section{Authors' contributions}

All authors gave substantial contributions to conception and design, drafting and critical revision of the manuscript. Research idea and study design: H-B G, J-Q P; data acquisition: K-K Z, G-Z Z, G-X S; data analysis/interpretation: W-H C, X-W; analysis: H-B G. All authors read and approved the final manuscript. 
Availability of data and materials

Data supporting the results are reported in this article and additional information is available.

\section{Competing interests}

Authors state no conflict of interest

\section{Ethics approval and consent to participate}

Not applicable.

\section{References}

[1] Alicic RZ, Rooney MT, Tuttle KR. Diabetic kidney disease: challenges, progress, and possibilities. Clin J Am Soc Nephrol. 2017;12:2032-45.

[2] Qi C, Mao X, Zhang Z, et al. Classification and differential diagnosis of diabetic nephropathy. J Diabetes Res. 2017;2017:8637138.

[3] Wang G, Ouyang J, Li S, et al. The analysis of risk factors for diabetic nephropathy progression and the construction of a prognostic database for chronic kidney diseases. J Transl Med. 2019;17:264.

[4] Zhou T, Sun L, Yang S, et al. 20(S)-Ginsenoside Rg3 Protects Kidney from Diabetic Kidney Disease via Renal Inflammation Depression in Diabetic Rats. J Diabetes Res. 2020;2020:7152176.

[5] Liu S, Ye L, Tao J, Ge C, et al. Total flavones of Abelmoschus manihot improve diabetic nephropathy by inhibiting the iRhom2/TACE signalling pathway activity in rats. Pharm Biol. 2017;56:1-11.

[6] Lai X, Tong D, Ai X, et al. Amelioration of diabetic nephropathy in $\mathrm{db} / \mathrm{db}$ mice treated with tibetan medicine formula Siwei Jianghuang Decoction Powder extract. Sci Rep. 2018;8:16707.

[7] Xu X, Li QJ, Xia S, Wang MM, Ji W. Tripterygium Glycosides for Treating Late-onset Rheumatoid Arthritis: A Systematic Review and Meta-analysis. Altern Ther Health Med. 2016;22:32-39.

[8] Ho LJ, Chang WL, Chen A, et al. Differential immunomodulatory effects by Tripterygium wilfordii Hook f-derived refined extract PG27 and its purified component PG490 (triptolide) in human peripheral blood T cells: potential therapeutics for arthritis and possible mechanisms explaining in part Chinese herbal theory "Junn-Chenn-Zuou-SS". J Transl Med. 2013;11:294.

[9] Ding Y, Zhang X, Ren X, et al. Traditional Chinese medicine versus regular therapy in Henoch-Schönlein purpura nephritis in children: study protocol for a randomized controlled trial. Trials. 2019;20:538.

[10] Wu W, Yang JJ, Yang HM, et al. Multi-glycoside of Tripterygium wilfordii Hook. f. attenuates glomerulosclerosis in a rat model of diabetic nephropathy by exerting anti-microinflammatory effects 
without affecting hyperglycemia. Int J Mol Med. 2017;40:721-730.

[11] Ren D, Zuo C, Xu G. Clinical efficacy and safety of Tripterygium wilfordii Hook in the treatment of diabetic kidney disease stage IV: A meta-analysis of randomized controlled trials. Medicine (Baltimore). 2019;98:e14604.

[12] Zheng XY. Guidelines for Clinical Research of New Chinese Medicine Drugs (trial). Beijing: China Medical Science Press; 2002. 156.

[13] Cai XP. ARB combined with Tripterygium glycosides in the treatment of diabetic nephropathy. J Clin Med Pract. 2012;16:112-4.

[14] Wang W. Different Doses of Tripterygium Glycosides in the Treatment of Diabetic Nephropathy: Effects on Blood Lipids. Kidney Blood Press Res. 2018;43:931-937.

[15] Nie CY, Chen LM, Chang BC, et al. The effect of tripterygium wilfordii on proteinuria in patients with diabetic nephropathy. Chinese Journal of Practical Internal Medicine. 2009;29:517-519.

[16] Chen H, Zhuang LP, Liu JF, et al. Effect of Irbesartan and Triptolide com bination on the level of urine protein in patients with diabetic nephropathy at high altitude area. Clinical Medicine of China. 2012,11:1149-1151.

[17] Song HX, Gong J, Chen W, et al. Effect of Triptol ide on Urinary Monocyte Chemottractant Protein-1 in Patients with Diabetic Nephropathy. Chinese Journal of Integrated Traditional and Western Medicine. 2005;5:416-418.

[18] Feng B, Ye ZL, Yang X, et al. Effect of triptolide on microinflammation in the patients with diabetic nephropathy. J Clin Nephrol, 2009,9(2):82-84.

[19] Guo YQ, Zuo YH. Clinical analysis of effect of Tripterysium glucosides in treatment of diabetic nephropathy. J Clin Nephrol. 2007;7:198-199.

[20] Fan HY, Shi YJ. Effect of Triptolide on Transforming Growth Factor $-\beta$ in Diabetic Nephropathy Cases. Chinese Journal of Integrated Traditional and Western Nephrology. 2005;7:395-397.

[21] Tu CF, Wang LJ, Gu LJ, et al. Effect of tripterygium wilfordii polyglycoside combined telmisartan on renal function and hemorheology in patients with diabetic nephropathy. Chinese Journal of General Practice. 2017;15:1527-1528+1595.

[22] Jiang X. Clinical observation of tripterygium glycosides combined with telmisartan in treatment 391 of diabetic nephropathy. Drugs \& Clinic. 2015;30:987-90. 392

[23] Wu YP, Shi NC. Clinical observation on the effect of Valsartan combined with Tripterygium glycosides

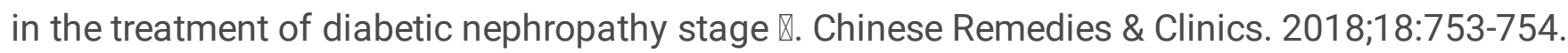


[24] Xu GB, Chen DJ, Chen WZ. Effect of Tripterygium Wilfordii Polyglycoside on Inflammatory FactorLevel in Patients with Diabetic Nephropathy, Chinese Archives of Traditional Chinese Medicine. 2017;35:2206-2208.

[25] Zhang HC. A clincial study of tripterygium glycosides tablets adjuvanttreatment of diabetic nephropathy during IV period. China Pharmaceuts. 2015;24:248-9.

[26] Su HJ, Qian Y, Li HS. Efficacy of tripterygium glycosides on proteinuria patients with diabetic nephropathy. Medical Journal of Wuhan University. 2013;34:296-298.

[27] Ge YC, Xie HL, Li SJ. Effect of tripterygium wilfordli in patients with diabetic nephropathy खa prospective randomized control clinical trial. J Nephrol Dialy Transplant. 2010;19:501-507+533.

[28] Wang ML, Zhang C. Clinical observation of Valsartan combined with Tripterygium wilfordii in the treatment of proteinuria in type 2 diabeticnephropathy. Chin J Clin Rational Drug Use. 2012;5:84-5.

[29] Jiang AL, Chu XY, Wang HT. Clinical observation on the effect of low-dose tripterygium glycosides on proteinuria in diabetic nephropathy. Chin J Integr Traditional West Nephrol. 2014;15:444-445.

[30] Tang ZH, Luo DW, Yuan WJ. A randomized controlled study of tripterygium glycosides polyglycoside in the treatment of diabetic nephropathy with moderate non-proliferative retinopathy. Chin $\mathrm{J}$ Integr Traditional West Nephrol. 2017;18:332-334.

[31] Zhao RY, Tang BS, Shi XL, et al. Clinical observation on 46 cases of diabetic nephropathy treated with Tripterygium wilfordii and Valsartan. Chin J Integr Traditional West Nephrol. 2011;12:811-3.

[32] Yin ZH, Xia Li. Clinical observation of tripterygium wilfordii polyglycoside on proteinuria in diabetic nephropathy. Lishizhen Medicine and Materia Medica Research. 2014;25:1676.

[33] Zheng XP. Clinical observation of tripterygium wilfordii on diabetic nephropathy. Journal of Clinical and Experimental Medicine. 2009;8:134-135.

[34] Ma RX, Zhao N, Zhang W. The effects and m echanism of tripterygium wilfordii Hook F com bination with irbesartan on urinary podocyte excretion in diabetic nephropathy patients. Chin J Intern Med. 2013;52:469-73.

[35] Chang X, Li L, Wang B, et al. Evaluation of the efficacy and safety of TWHF in diabetic nephropathy patients with overt proteinuria and normal eGFR. J Formos Med Assoc. 2020;119:685-692.

[36] Ma RX, Xu Y, Jiang W, et al. Combination of Tripterygium wilfordii Hook F and angiotensin receptor blocker synergistically reduces excretion of urinary podocytes in patients with type 2 diabetic kidney disease. Biotechnol Biotechnol Equip. 2015;29:139-146. 
[37] Gao SP. A randomized controlled study of Tripterygium wilfordii polyglycoside in the treatment of diabetic nephropathy. Hainan Medical Journal. 2012;23:31-32.

[38] Zheng YH. Tripterygium wilfordii clinical research for the treatment of diabetic nephropathy stage IV[Master]: Guangzhou University of Chinese Medicine; 2014.

[39] Ringholm L, Damm JA, Vestgaard M, et al. Mathiesen ER. Diabetic Nephropathy in Women With Preexisting Diabetes: From Pregnancy Planning to Breastfeeding. Curr Diab Rep. 2016;16(2):12.

[40] Yamahara K, Yasuda M, Kume S, Koya D, Maegawa H, Uzu T. The role of autophagy in the pathogenesis of diabetic nephropathy. J Diabetes Res. 2013;2013:193757.

[41] Hong Y, Gui Z, Cai X, et al. Clinical efficacy and safety of tripterygium glycosides in treatment of stage IV diabetic nephropathy: A meta-analysis. Open Med (Wars). 2016 Dec 30;11(1):611-617.

[42] Abdelhafiz AH, Nahas ME, de Oliveira JM. Management of diabetic nephropathy in older patients: a need for flexible guidelines. Postgrad Med. 2014 Jul;126(4):171-7.

[43] Huang YR, Wan YG, Sun W, et al. Effects and mechanisms of multi-glycoside of Tripterygium wilfordii improving glomerular inflammatory injury by regulating p38MAPK signaling activation in diabetic nephropathy rats. Zhongguo Zhong Yao Za Zhi. 2014;39(21):4102-9. Chinese.

[44] Wang S, Li R, He S,et al. Tripterygium wilfordii Glycosides Upregulate the New Anti-Inflammatory Cytokine IL-37 through ERK1/2 and p38 MAPK Signal Pathways. Evid Based Complement Alternat Med. 2017;2017:9148523.

[45] Fang JY, Yang Y, Zhang Z, Jiang SM, Yu TY, Li WG. Effects of adding tripterygium glycosides to angiotensin-converting enzyme inhibitors or angiotensin receptor blockers on albuminuria in patients with diabetic nephropathy. Chronic Dis Transl Med. 2020 Feb 10;6(1):18-26.

[46] Wu X, Huang Y, Zhang Y, et al. Efficacy of tripterygium glycosides combined with ARB on diabetic nephropathy: a meta-analysis. Biosci Rep. 2020 Nov 27;40(11):BSR20202391.

\section{Figures}




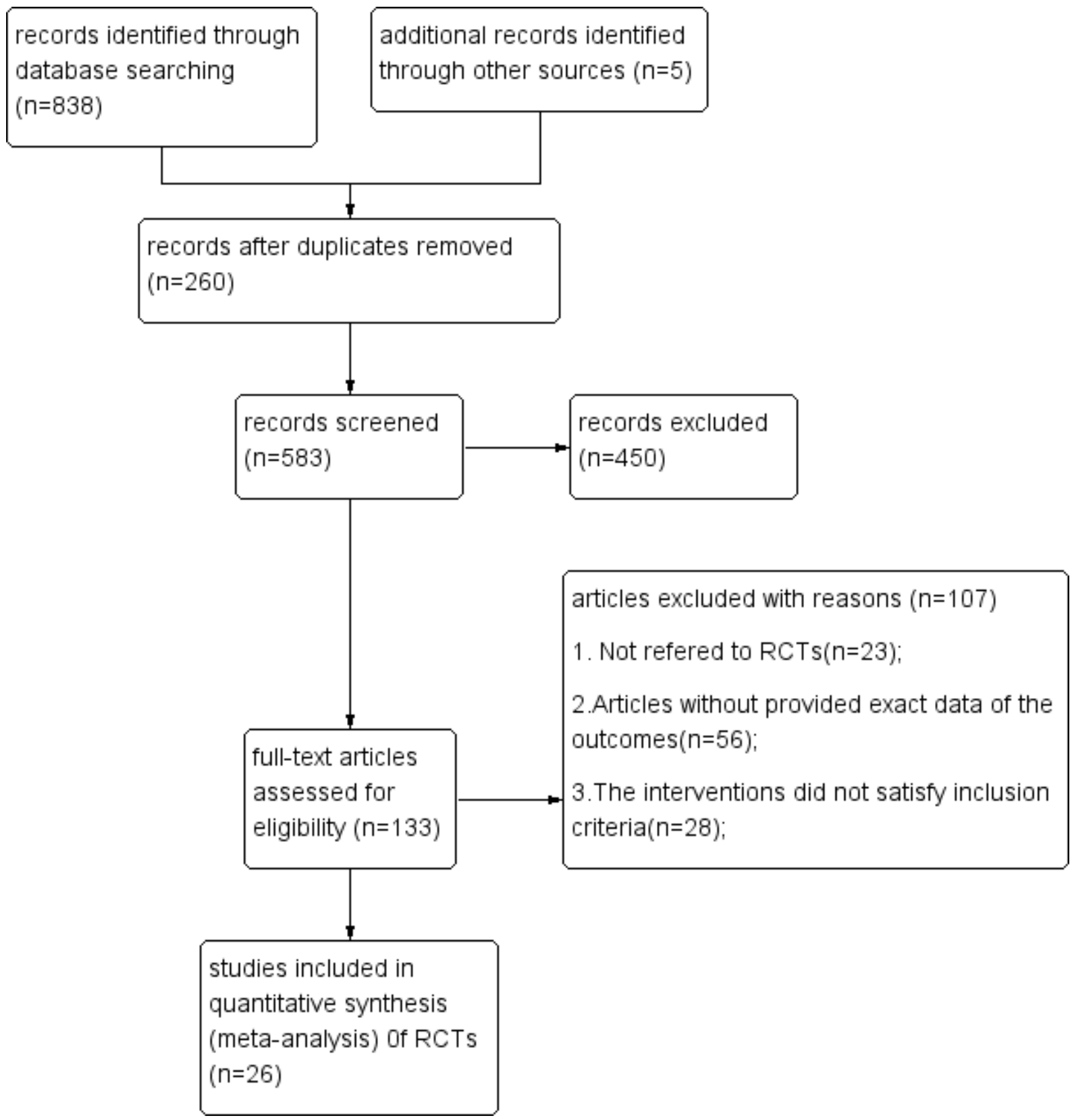

\section{Figure 1}

Flow diagram of study identification process 
Random sequence generation (selection bias) Allocation concealment (selection bias)

Blinding of participants and personnel (performance bias)

Blinding of outcome assessment (detection bias)

Incomplete outcome data (attrition bias)

Selective reporting (reporting bias)

Other bias
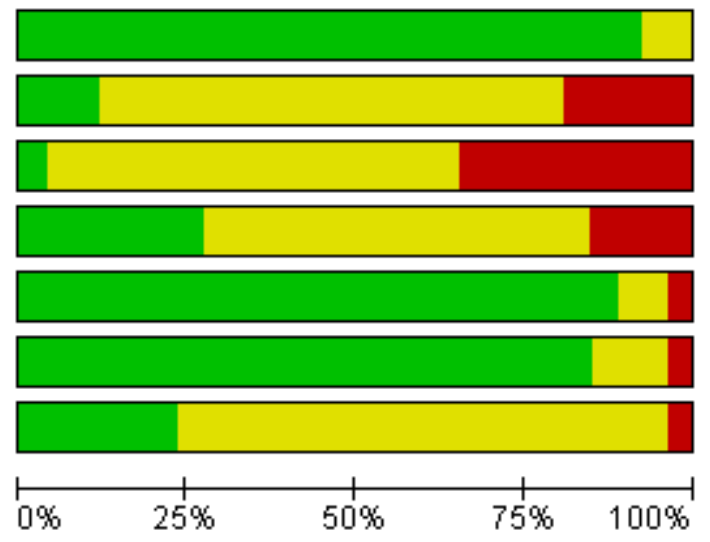

Low risk of bias

Unclear risk of bias

High risk of bias

Figure 2

Risk of bias graph: Each risk of bias item was included for each study. 


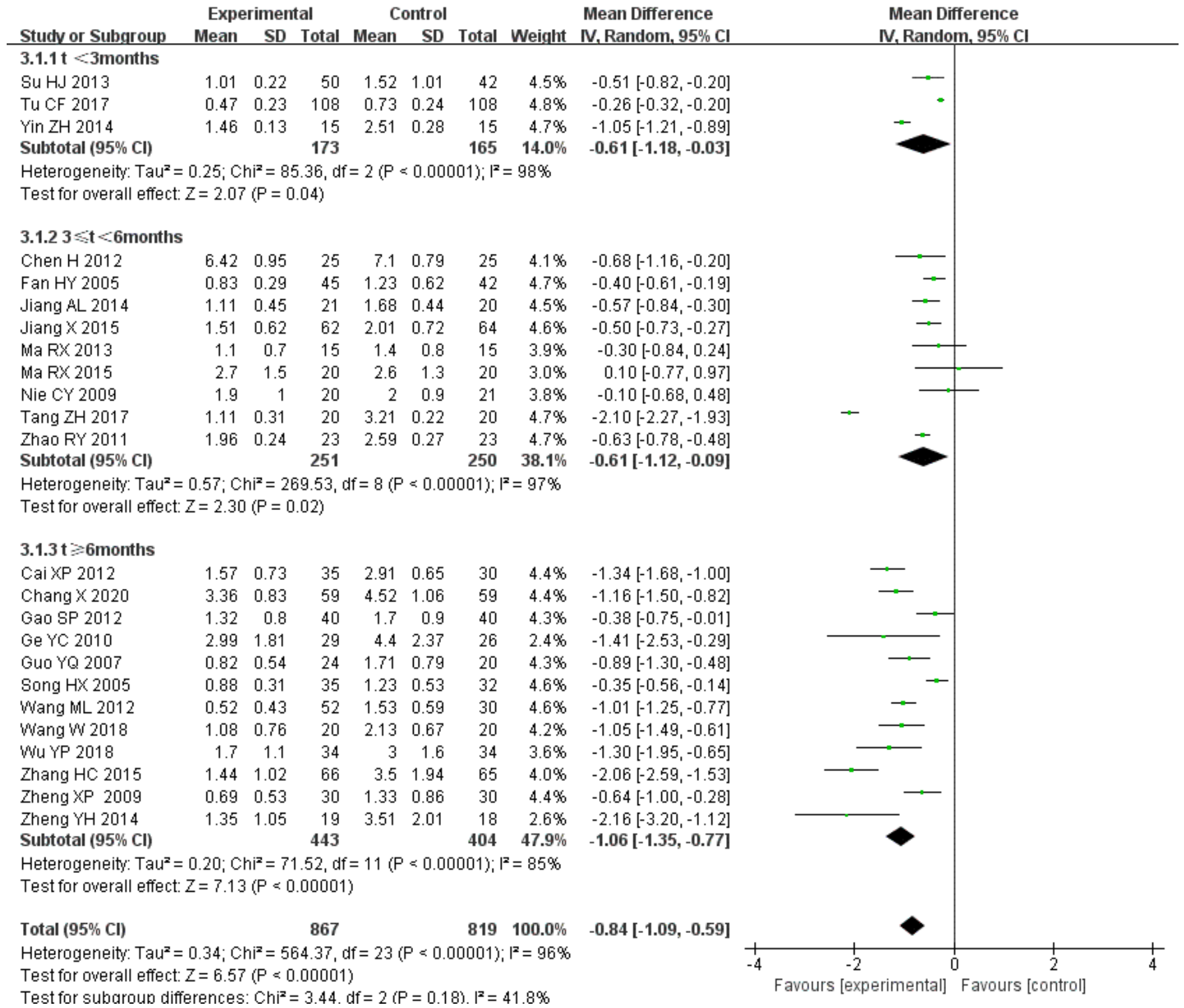

\section{Figure 3}

Subgroup analysis of TGs in the treatment of DN based on the course of treatment, outcome: 24h TUP. 


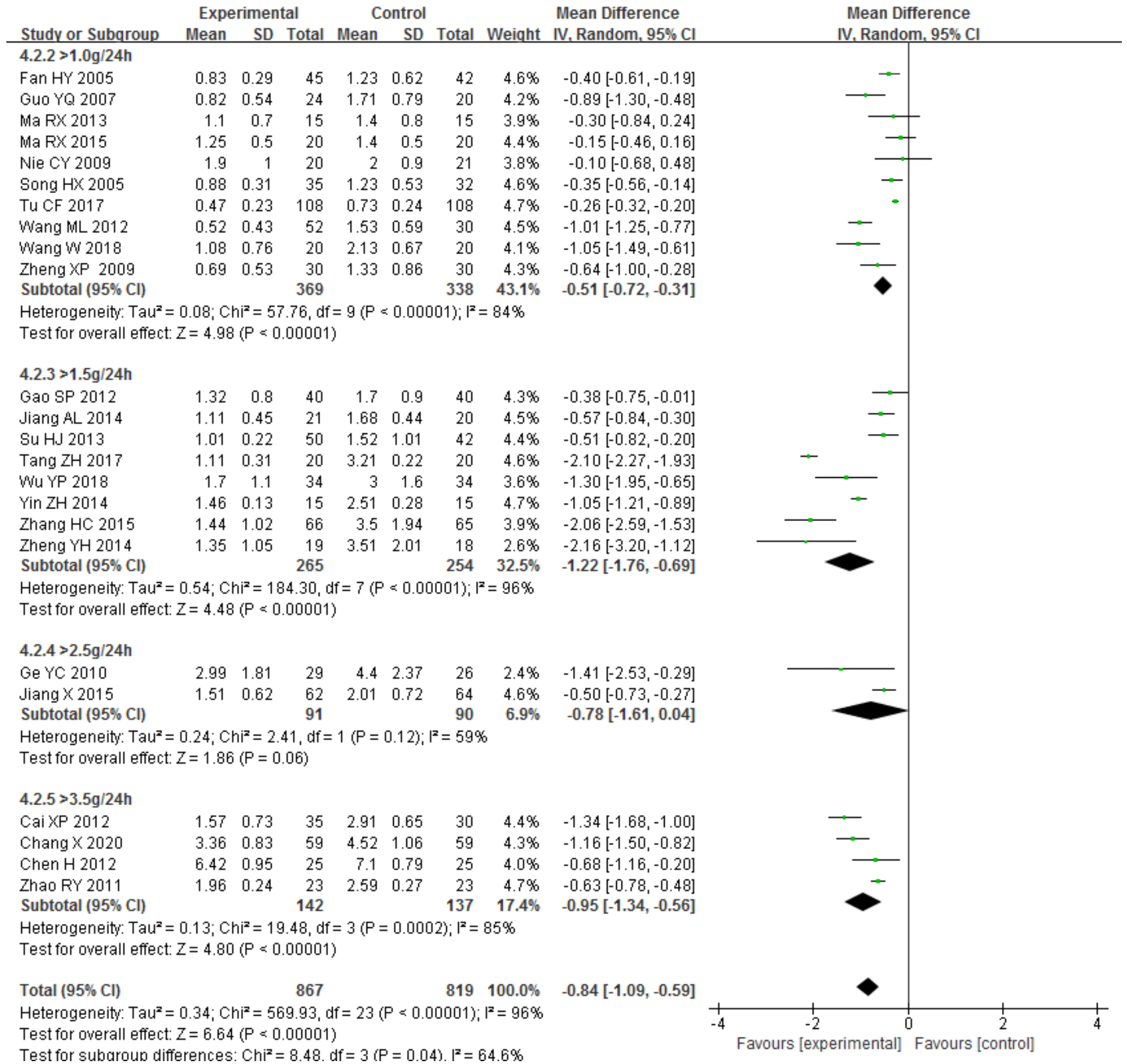

\section{Figure 4}

Subgroup analysis of TGs in the treatment of DN based on the 24h UTP baseline of inclusion criteria, outcome: 24h UTP 


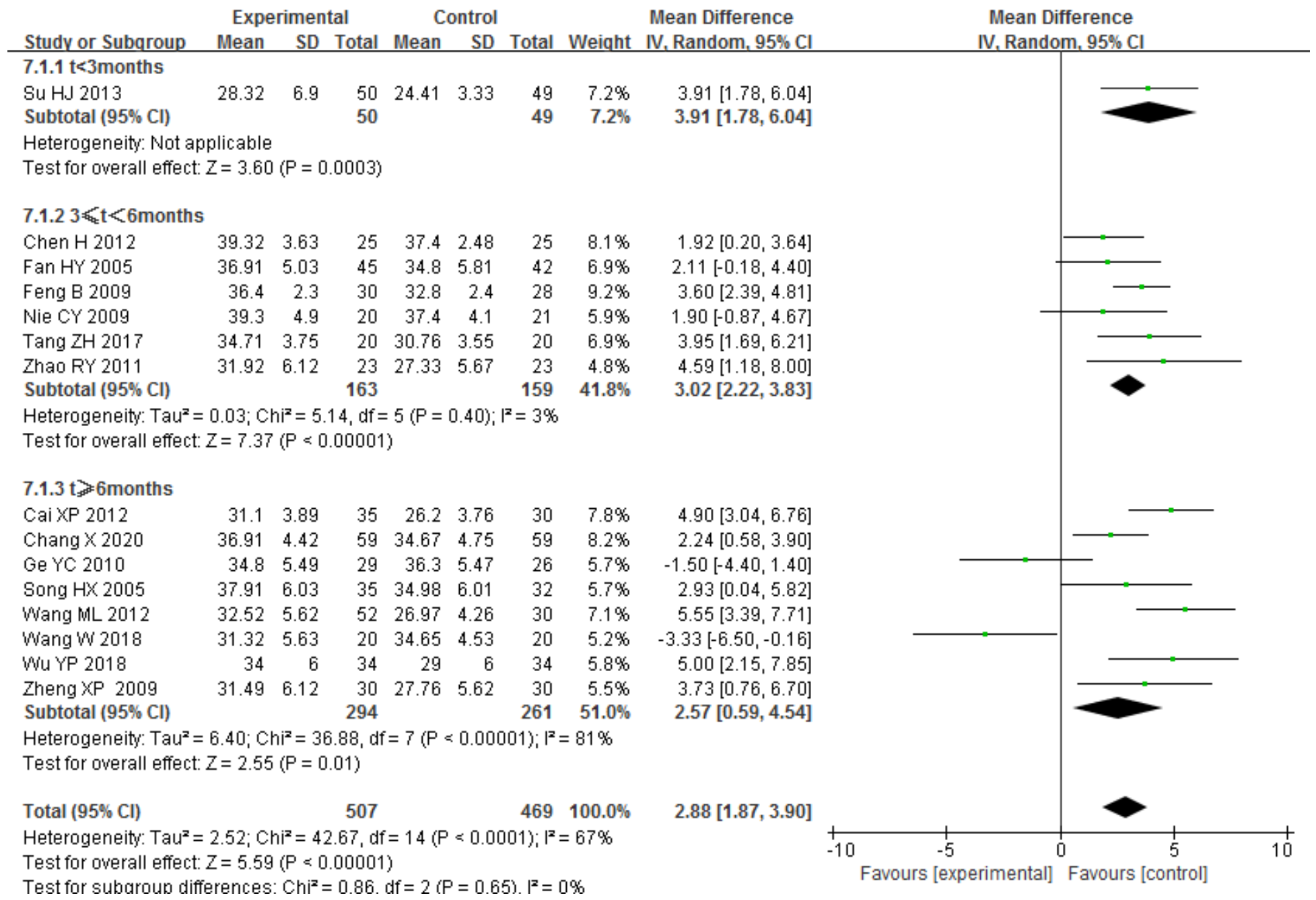

\section{Figure 5}

Subgroup analysis of TGs in the treatment of DN based on the course of treatment, outcome: Alb. 


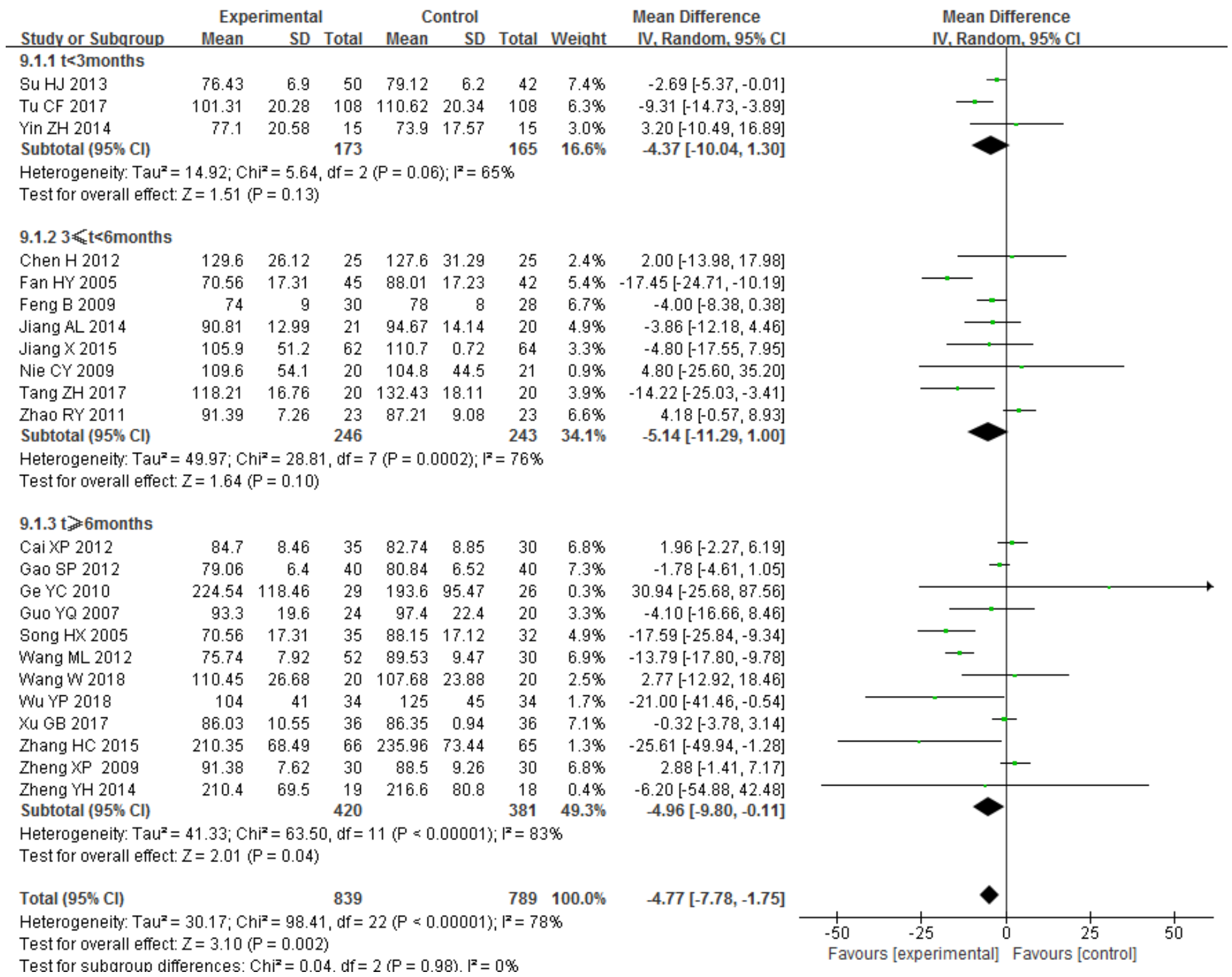

\section{Figure 6}

Subgroup analysis of TGs in the treatment of DN based on the course of treatment, outcome: SCr.

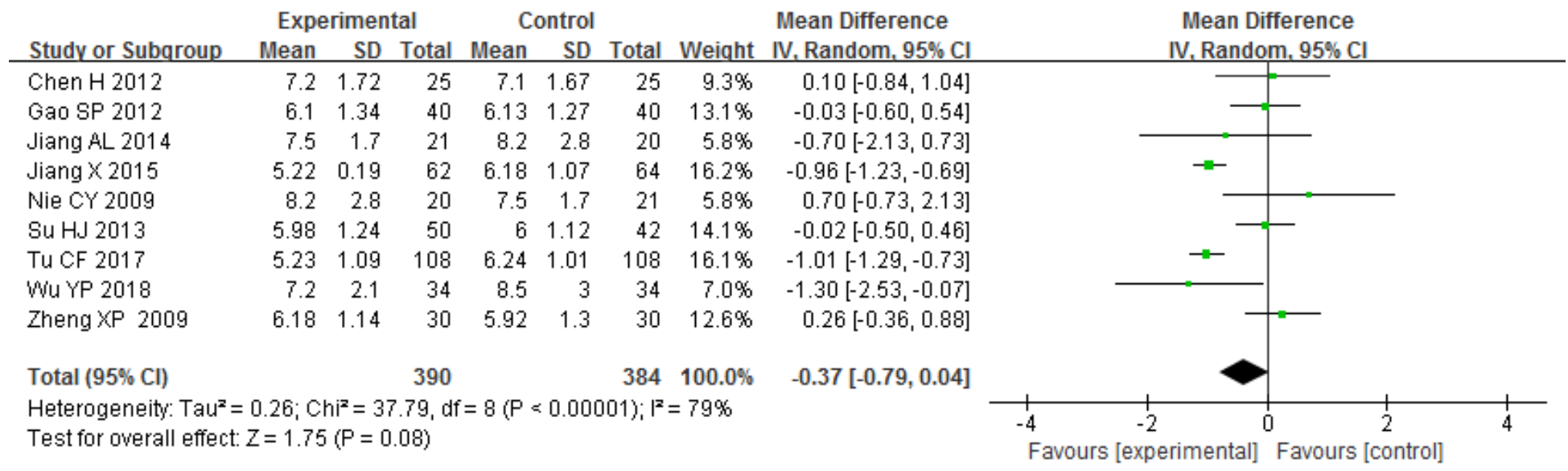

\section{Figure 7}


Forest plots of TGs in the treatment of DN, outcome: BUN.

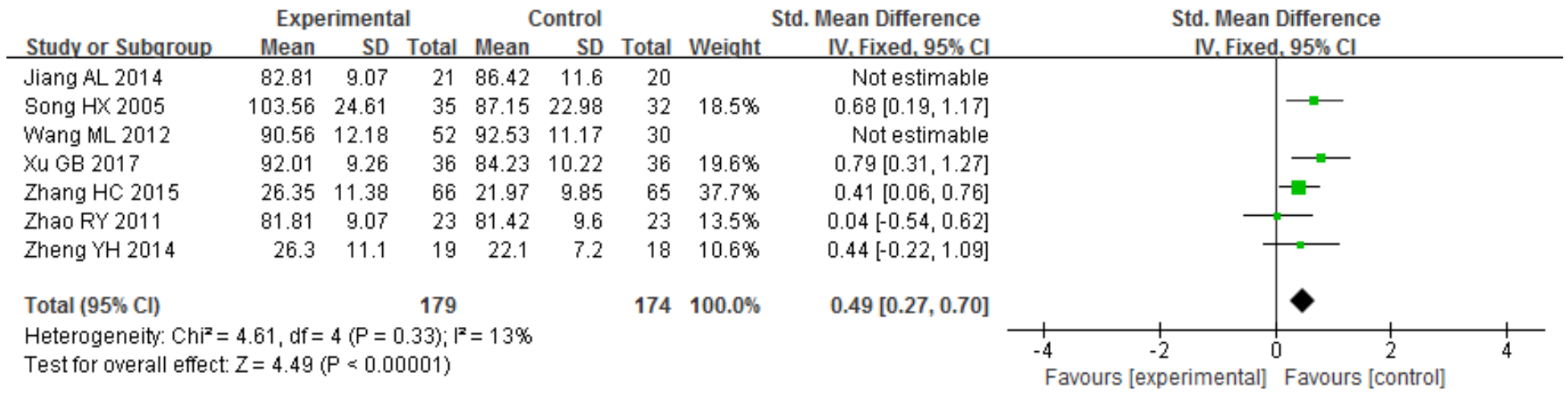

Figure 8

Forest plots of TGs in the treatment of DN, outcome: $\mathrm{CCr}$.

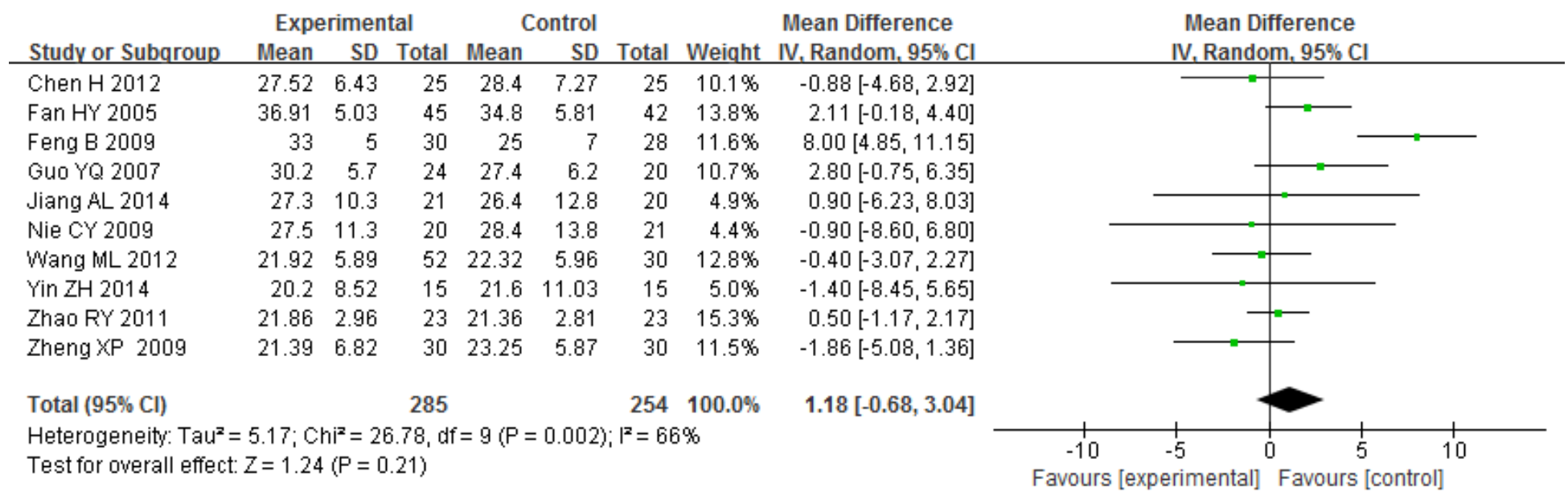

\section{Figure 9}

Forest plots of TGs in the treatment of DN, outcome: ALT.

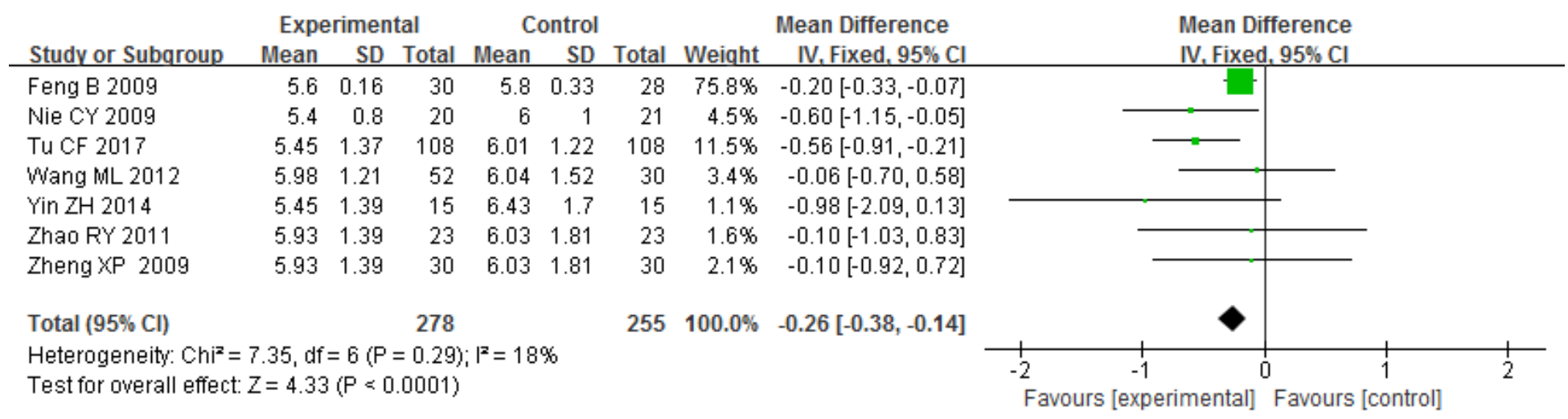

\section{Figure 10}

Forest plots of TGs in the treatment of DN, outcome: WBC. 


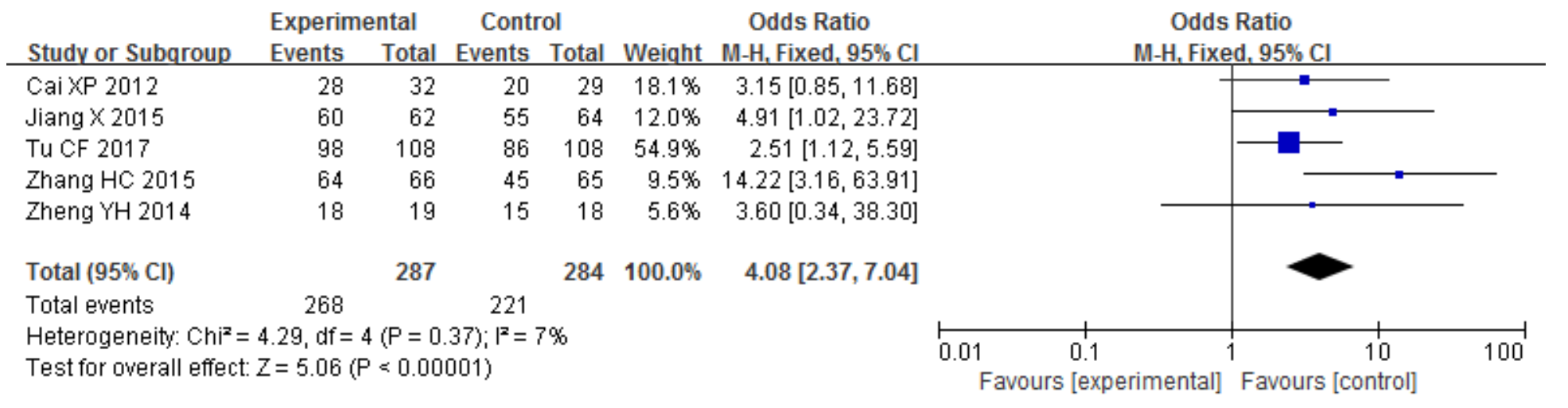

\section{Figure 11}

Forest plots of the total efficiency of TGs in the treatment of DN

\section{Supplementary Files}

This is a list of supplementary files associated with this preprint. Click to download.

- PRISMA2009checklist.doc

- Supplementaryinformation.docx 